\title{
Mathematical Modelling of Reduced Order Induction Machines for VFT Applications
}

\author{
Edgar Lucas \\ Department of Electronic \\ and Electrical Engineering \\ University of Strathclyde \\ Glasgow, UK \\ edgar.lucas@strath.ac.uk
}

\author{
David Campos-Gaona \\ Department of Electronic \\ and Electrical Engineering \\ University of Strathclyde \\ Glasgow, UK \\ d.campos-gaona@strath.ac.uk
}

\author{
Olimpo Anaya-Lara \\ Department of Electronic \\ and Electrical Engineering \\ University of Strathclyde \\ Glasgow, UK \\ olimpo.anaya-lara@strath.ac.uk
}

\begin{abstract}
This paper presents a reduced order approach to the modelling of induction machines for the purposes of power transfer between asynchronous networks. The method of interconnection between the networks under consideration is a variable frequency transformer (VFT) whose technology is rooted in that of an induction machine. Attempts to apply a high-order model to VFT studies have been met with numerical instabilities and unacceptably long computation times. A reduced order approach therefore serves to reduce the complexity of the simulation by neglecting transient behaviour. The classical fifth order method is presented, upon which a third order and first order model are derived. These differ from the traditional reduced order models due to the inclusion of an otherwise neglected frequency differential term. Care has also been taken to avoid algebraic loops during simulation so that additional delays need not be introduced. The performance of the models is then analysed when they are operating as anduction machine and then as a VFT for asynchronous power transfer simulations. The result is a stable, low-order model that performs correctly under a wide range of VFT operative scenarios, including asynchronous and different-frequency networks connected to its terminals.
\end{abstract}

\section{INTRODUCTION}

A new concept that facilities the controllable, bi-directional power flow between two asynchronous networks has emerged in the form of the VFT [1]. Traditionally, the transfer of power between two grids with different frequencies has been accomplished through the use of phase shifting transformers and back-to-back high-voltage direct-current (B2B-HVDC) converters, however each of these solutions has its drawbacks [2].

The construction of the VFT is similar to that of a rotary transformer. Three-phase windings are present on both the stator and the rotor allowing the connection of two independent networks. A DC drive motor is connected to the rotor to adjust the phase angle difference between rotor and stator. Neglecting resistance, power flow through the VFT is given by (1) and is proportional to the angle of the rotary transformer which in turn is proportional to applied torque.

$$
P_{V F T}=\frac{V_{s} V_{r}}{X_{s r}} \sin \left(\theta_{n e t}\right)
$$

Where $P_{V F T}$ is the power flow through the VFT, $V_{s}$ and $V_{r}$ are the RMS voltages at the stator and rotor terminals respectively, $X_{s r}$ is the total reactance between stator and rotor terminals and $\theta_{\text {net }}$ is the net phase angle between stator and rotor. If a positive torque is applied to the shaft, power will flow from stator to rotor. If torque is applied in the opposite direction, power flow will reverse and move from rotor to stator. If the two systems are not in synchronism, the rotor will rotate constantly at a speed proportional to the slip given by (2), where $f_{s}, f_{r}$ are the frequencies of the stator and rotor respectively. Load flow is maintained during this operation despite the drifting frequencies [3].

$$
\operatorname{slip}=\frac{f_{s}-f_{r}}{f_{s}}
$$

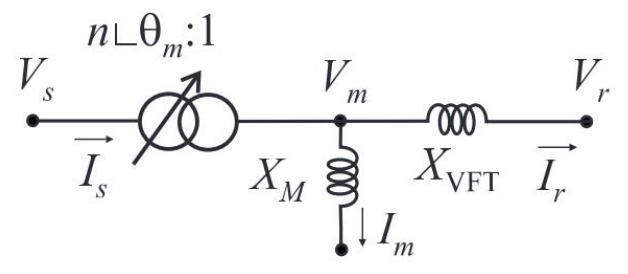

Fig. 1. Phase angle regulator representation of VFT [4]

Previous studies have modelled the VFT as a phase angle regulator where the VFT is simulated by a reactance $X_{V F T}$, a magnetising branch $X_{m}$ and an ideal transformation representing the phase shift as shown in fig. I. This method is detailed in the literature [4] - [7] and is mainly used for steady state power flow studies. This paper explores an 
alternative approach to VFT simulation using reduced order methods which have been specifically designed to preserve all frequency characteristics and also to avoid algebraic loops during simulation. Benefits of this method include the addition of winding resistances, the possibility to include turns ratios into the machine and the ability to perform steady state simulations with reduced computational effort.

\section{II. $5^{\text {th }}$ ORDER MODEL}

The 5th order model is obtained through the use of a directquadrature (dq) axis transformation from the unsimplified abc line elements model. This is well documented in literature [11] but is briefly presented here for completion. Ignoring the zero-sequence elements, the electrical system of an induction generator is fully represented by the dynamic equations in (3), where $v, i, r, \lambda$ and $\omega$ stand for voltage, current, resistance, flux linkage and angular frequency respectively. The subscripts $s, r$ denote stator or rotor quantities while the subscripts $d, q$ denote the $\mathrm{d}$ and $\mathrm{q}$ axes components. All rotor quantities shall be referred to the stator, negating the need for primed variables in the derivations.

$$
\begin{aligned}
& v_{d s}=i_{d s} r_{s}+\frac{d \lambda_{d s}}{d t}-\omega_{s} \lambda_{q s} \\
& v_{q s}=i_{q s} r_{s}+\frac{d \lambda_{q s}}{d t}+\omega_{s} \lambda_{d s} \\
& v_{d r}=i_{d r} r_{r}+\frac{d \lambda_{d r}}{d t}-\left(\omega_{s}-\omega_{r}\right) \lambda_{q r} \\
& v_{q r}=i_{q r} r_{r}+\frac{d \lambda_{q r}}{d t}+\left(\omega_{s}-\omega_{r}\right) \lambda_{d r}
\end{aligned}
$$

These equations account for four of the orders. The fifth order comes from the mechanical system given in (4), where $J$, $T_{\text {em }}, T_{\text {load }}, T_{\text {damp }}$ are the machine's inertia; electromechanical torque; load torque and damping torque respectively. For simplicity, damping torque will be assumed to be zero.

$$
\frac{d \omega_{r}}{d t}=\frac{1}{J}\left(T_{e m}-T_{l o a d}-T_{d a m p}\right)
$$

Electromechanical torque can be expressed in a number of ways based on what is convenient as in (5). Here, $P$ is the number of poles, not to be confused with pole-pairs.

$$
\begin{aligned}
T_{e m} & =\left(\frac{P}{2}\right)\left(\frac{3}{2}\right)\left(i_{d r} \lambda_{q r}-i_{q r} \lambda_{d r}\right) \\
& =\left(\frac{P}{2}\right)\left(\frac{3}{2}\right)\left(i_{q s} \lambda_{d s}-i_{d s} \lambda_{q s}\right) \\
& =\left(\frac{P}{2}\right)\left(\frac{3}{2}\right) L_{m}\left(i_{q s} i_{d r}-i_{d s} i_{q r}\right)
\end{aligned}
$$

Substituting equations (6) - (8) into (3) and rearranging into integral form for use in simulation, the full system is described by (9),

$$
\begin{aligned}
i_{d s} & =\frac{\lambda_{d s}-\lambda_{d m}}{L_{l s}} & i_{d r} & =\frac{\lambda_{d r}-\lambda_{d m}}{L_{l r}} \\
i_{q s} & =\frac{\lambda_{q s}-\lambda_{q m}}{L_{l s}} & i_{q r} & =\frac{\lambda_{q r}-\lambda_{q m}}{L_{l r}}
\end{aligned}
$$

where

$$
\begin{aligned}
& \lambda_{d m}=L_{M}\left(\frac{\lambda_{d s}}{L_{l s}}+\frac{\lambda_{d r}}{L_{l r}}\right) \\
& \lambda_{q m}=L_{M}\left(\frac{\lambda_{q s}}{L_{l s}}+\frac{\lambda_{q r}}{L_{l r}}\right)
\end{aligned}
$$

and

$$
L_{M}=\frac{1}{\left[\frac{1}{L_{l s}}+\frac{1}{L_{l r}}+\frac{1}{L_{m}}\right]}
$$

$L_{l s}, L_{l r}, L_{m}$ are the stator leakage flux, rotor leakage flux and mutual flux respectively and $L_{M}$ is a common factor in the derivation.

$$
\begin{aligned}
\lambda_{d s} & =\int\left(v_{d s}-\frac{r_{s}}{L_{l s}}\left(\lambda_{d s}-\lambda_{d m}\right)+\omega_{s} \lambda_{q s}\right) \mathrm{d} t \\
\lambda_{q s} & =\int\left(v_{q s}-\frac{r_{s}}{L_{l s}}\left(\lambda_{q s}-\lambda_{q m}\right)-\omega_{s} \lambda_{d s}\right) \mathrm{d} t \\
\lambda_{d r} & =\int\left(v_{d r}-\frac{r_{s}}{L_{l s}}\left(\lambda_{d r}-\lambda_{d m}\right)+\left(\omega_{s}-\omega_{r}\right) \lambda_{q r}\right) \mathrm{d} t \\
\lambda_{q r} & =\int\left(v_{q r}-\frac{r_{s}}{L_{l s}}\left(\lambda_{q r}-\lambda_{q m}\right)-\left(\omega_{s}-\omega_{r}\right) \lambda_{d r}\right) \mathrm{d} t \\
\omega_{r} & =\frac{1}{J} \int\left(T_{\text {em }}-T_{\text {load }}\right) \mathrm{d} t
\end{aligned}
$$

Given its high order, the 5th order model is able to faithfully reproduce most of the steady and transient state behaviour of a VFT for asynchronous and different-frequency grids. However given its extended computational requirements, it is seldom used in most of the VFT analysis presented in literature. The possibility of using low-order models specifically designed for the operative conditions of the VFT is analysed in sections III and IV.

\section{III. $3^{\text {rd }}$ ORDER MODEL}

It is possible to reduce the order of the model and thus reduce the complexity of the simulation by neglecting the stator transient terms [8]. This is equivalent to setting the differential terms to zero, as in (10). In the traditional derivation of low order models of induction machines, the stator frequency is assumed constant so that a $\frac{d \omega_{s}}{d t}$ term may also be removed, however this may not be valid in VFT applications operating at different frequencies. One method to include this frequency characteristic is addressed in [10].

An alternative derivation presented below not only preserves the frequency characteristics of the system but also 
serves to avoid any algebraic loops within the simulation. The layout of the equations are such that they do not require any additional delays to be brought into the model as everything is solved sequentially. An added benefit arises due to the similarities in equations for the 5 th and 3rd order models. Rotor flux remains as the state variable so there is no pre or post-processing required to convert back into usable quantities.

With stator transients ignored, the equations describing the stator electrical system in (10) can be solved simultaneously such that they are defined by voltages and rotor quantities.

$$
\begin{aligned}
& v_{d s}-i_{d s} r_{s}+\omega_{s} \lambda_{q s}=0 \\
& v_{q s}-i_{q s} r_{s}-\omega_{s} \lambda_{d s}=0
\end{aligned}
$$

Substituting (6) and (8) into (10),

$$
v_{d s}-\frac{r_{s}}{L_{l s}}\left[\lambda_{d s}-\frac{\left(\frac{\lambda_{d s}}{L_{l s}}+\frac{\lambda_{d r}}{L_{l r}}\right)}{\left(\frac{1}{L_{l s}}+\frac{1}{L_{l r}}+\frac{1}{L_{m}}\right)}\right]+\omega_{s} \lambda_{q s}=0
$$

Rearranging for $\lambda_{d s}$ and substituting for the stator and rotor self-inductances, $L_{s s}=L_{m}+L_{l s}$ and $L_{r r}=L_{m}+L_{l r}$ :

$$
\lambda_{d s}=\frac{\lambda_{q s} \omega_{s}}{r_{s}}\left(L_{s s}-\frac{L_{m}^{2}}{L_{r r}}\right)+\frac{v_{d s}}{r_{s}}\left(L_{s s}-\frac{L_{m}^{2}}{L_{r r}}\right)+\frac{L_{m} \lambda_{d r}}{L_{r r}}
$$

Making the substitution $\left(L_{s s}-\frac{L_{m}^{2}}{L_{r r}}\right)=L$ gives (13).

$$
\lambda_{d s}=\frac{v_{d s} L}{r_{s}}+\frac{L_{m} \lambda_{d r}}{L_{r r}}+\frac{\lambda_{q s} \omega_{s} L}{r_{s}}
$$

similarly,

$$
\lambda_{q s}=\frac{v_{q s} L}{r_{s}}+\frac{L_{m} \lambda_{q r}}{L_{r r}}-\frac{\lambda_{d s} \omega_{s} L}{r_{s}}
$$

To avoid algebraic loops in the simulation process, these equations are solved simultaneously to give (15). The stator fluxes are now defined only in terms of rotor quantities and applied voltages.

$$
\begin{aligned}
\lambda_{d s} & =\frac{L_{r r} L r_{s} v_{d s}+L_{r r} L^{2} \omega_{s} v_{q s}+L_{m} r_{s}^{2} \lambda_{d r}+L_{m} L r_{s} \omega_{s} \lambda_{q r}}{\left(L^{2} \omega_{s}^{2}+r_{s}^{2}\right) L_{r r}} \\
\lambda_{q s} & =\frac{L_{r r} L r_{s} v_{q s}-L_{r r} L^{2} \omega_{s} v_{d s}+L_{m} r_{s}^{2} \lambda_{q r}-L_{m} L r_{s} \omega_{s} \lambda_{d r}}{\left(L^{2} \omega_{s}^{2}+r_{s}^{2}\right) L_{r r}}
\end{aligned}
$$

These can be further simplified by noting that under the prescribed axis alignment, $v_{q s}=0$. Therefore,

$$
\begin{aligned}
\lambda_{d s} & =\frac{L_{r r} L r_{s} v_{d s}+L_{m} r_{s}^{2} \lambda_{d r}+L_{m} L r_{s} \omega_{s} \lambda_{q r}}{\left(L^{2} \omega_{s}^{2}+r_{s}^{2}\right) L_{r r}} \\
\lambda_{q s} & =\frac{-L_{r r} L^{2} \omega_{s} v_{d s}+L_{m} r_{s}^{2} \lambda_{q r}-L_{m} L r_{s} \omega_{s} \lambda_{d r}}{\left(L^{2} \omega_{s}^{2}+r_{s}^{2}\right) L_{r r}}
\end{aligned}
$$

The algebraic equations in (16) can be now be used together with the remaining three differential equations to define the third order model of an induction machine.

$$
\begin{aligned}
\lambda_{d r} & =\int\left(v_{d r}-\frac{r_{s}}{L_{l s}}\left(\lambda_{d r}-\lambda_{d m}\right)+\left(\omega_{s}-\omega_{r}\right) \lambda_{q r}\right) \mathrm{d} t \\
\lambda_{q r} & =\int\left(v_{q r}-\frac{r_{s}}{L_{l s}}\left(\lambda_{q r}-\lambda_{q m}\right)-\left(\omega_{s}-\omega_{r}\right) \lambda_{d r}\right) \mathrm{d} t \\
\omega_{r} & =\frac{1}{J} \int\left(T_{e m}-T_{m e c h}\right) \mathrm{d} t
\end{aligned}
$$

\section{IV. $1^{\text {st }}$ ORDER MODEL}

The model can be further simplified by neglecting the rotor dynamics as well as the stator dynamics. The resulting model would obviously not be useful for electrical transient studies but the benefits in computational speed would become apparent for power flow studies. As was the case in the previous section, the following derivation does not neglect any frequency terms such that what is presented is a completely unsimplified solution which also does not require any additional delays to be introduced into the model.

By setting the rotor flux differential terms equal to zero then the equations of the rotor dynamics turn to be as in (18).

$$
\begin{aligned}
& v_{d r}-i_{d r} r_{r}+\left(\omega_{s}-\omega_{r}\right) \lambda_{q r}=0 \\
& v_{q r}-i_{q r} r_{r}-\left(\omega_{s}-\omega_{r}\right) \lambda_{d r}=0
\end{aligned}
$$

One downside to this approach is that as the order of the system decreases, the length of the defining equations increases exponentially. This can be seen in the previous derivation of the 3rd order model and becomes much more cumbersome as we progress to the 1st order. The system of equations must be manipulated to depend only on the voltage inputs and rotor speed as this is the only state variable.

Substituting (6) and (7) into (18), the rotor voltage equation becomes:

$v_{d r}-\frac{r_{r}}{L_{l r}}\left[\lambda_{d r}-\frac{\left(\frac{\lambda_{d r}}{L_{l r}}+\frac{\lambda_{d s}}{L_{l s}}\right)}{\left(\frac{1}{L_{l r}}+\frac{1}{L_{l s}}+\frac{1}{L_{m}}\right)}\right]+\left(\omega_{s}-\omega_{r}\right) \lambda_{q r}=0$ 
Rearranging in terms of $\lambda_{d r}$ and substituting for stator flux in (15) previously derived in the $3 \mathrm{rd}$ order system we arrive at an expression for rotor flux in terms of $\omega_{r}$. Note that in order to make the expression more manageable, the substitutions in (20) are applied.

$$
\begin{aligned}
A= & \left(L_{l r} L_{l s}+L_{l r} L_{m}+L_{l s} L_{m}\right)\left(L^{2} \omega_{s}^{2}+r_{s}^{2}\right) \\
B= & \left(L_{l s} L_{r r}-L_{m}^{2}+L_{m} L_{r r}\right) r_{s}^{2} \\
& +\left(L_{l s} L_{r r}+L_{m} L_{r r}\right) L^{2} \omega_{s}^{s}
\end{aligned}
$$

Similarly by applying the same process to the q-axis, the system of simultaneous equations in (21) is obtained. It can be proved analytically that these two equations when solved produce the desired result.

$$
\begin{aligned}
\lambda_{d r}= & \frac{\lambda_{q r}\left(L L_{m}^{2} r_{s} r_{r} \omega_{s}+A L_{r r}\left(\omega_{s}-\omega_{r}\right)\right)}{B r_{r}} \\
& +\frac{L L_{r r} L_{m}\left(L v_{q s} \omega_{s}+r_{s} v_{d s}\right)}{B}+\frac{v_{d r} L_{r r} A}{B r_{r}} \\
\lambda_{q r}=- & \frac{\lambda_{d r}\left(L L_{m}^{2} r_{s} r_{r} \omega_{s}-A L_{r r}\left(\omega_{s}-\omega_{r}\right)\right)}{B r_{r}} \\
& -\frac{L L_{r r} L_{m}\left(L v_{d s} \omega_{s}-r_{s} v_{q s}\right)}{B}+\frac{v_{q r} L_{r r} A}{B r_{r}}
\end{aligned}
$$

The only remaining differential equation is now that of the rotor speed $\omega_{r}$, with all other variables being defined in terms of this one state variable.

$$
\omega_{r}=\frac{1}{J} \int\left(T_{e m}-T_{m e c h}\right) \mathrm{d} t
$$

\section{Simulations}

The following simulations were computed in Simulink using a step time of $200 \mu$ s and simulation times of 400 s with the ode45 solver and 'accelerator' mode enabled. The parameters are given by their respective tables in the following section along with the voltages of the stator and rotor side network (where appropriate), depending on the type of machine under test. The frequency is set at $50 \mathrm{~Hz}$ unless otherwise stated.

\section{A. Performance as an induction machine}

The performance of the developed models in previous sections will now be analysed for comparison and to asses their suitability in different applications. Firstly, a no-load test is conducted to check that they function accurately as induction machines. The machine parameters for a $2 \mathrm{MW}$ machine are given in table I, where $S_{b}$ is the rated power of the machine and $\mathrm{H}$ is the per-unit inertia constant.

In this test $T_{\text {load }}$ is set to zero and the rotor windings are short circuited. When the stator is energised with a three phase source the rotor quickly accelerates to $1 p u$ as $T_{e m}$
TABLE I

INDUCTION MACHINE PARAMETERS

\begin{tabular}{l|l|l|l} 
Parameter & Value & Parameter & Value \\
\hline$S_{b}$ & $2(\mathrm{MW})$ & $L_{l s}$ & $0.09241(\mathrm{pu})$ \\
$V_{s}$ & $690(\mathrm{~V})$ & $L_{l r}$ & $0.09955(\mathrm{pu})$ \\
$r_{s}$ & $0.00488(\mathrm{pu})$ & $L_{m}$ & $3.935(\mathrm{pu})$ \\
$r_{r}$ & $0.00549(\mathrm{pu})$ & $H$ & $3.5(\mathrm{~s})$
\end{tabular}

approaches zero where they both remain in a steady-state. At $t=250 \mathrm{~s}$, a torque of $-1 \mathrm{pu}$ is applied to the shaft, simulating generator action. At $t=300 \mathrm{~s}$ this torque is removed and at $t=350 \mathrm{~s}$ a positive torque is applied, simulating a load. Shown in fig. 2 we see the machines originally at steady-state and then following the prescribed trajectories dictated by the applied torque. The models are all in good agreement with other, although the transient behaviour differs as one would expect. Fig. 3 shows a close-up of the transient, highlighting the smooth response of the first order model in comparison with the oscillatory motion of the 5th and 3rd orders.

Table II gives the computation times for each model. The 3 rd and 1 st order models show a $20 \%$ and a $27 \%$ decrease in elapsed time respectively when compared to the fifth order.

TABLE II

SIMULATION TIMES OF INDUCTION MACHINE MODELS

\begin{tabular}{l|l} 
Model & Time $(\mathrm{s})$ \\
\hline 5th Order & 15.80 \\
3rd Order & 12.49 \\
1st Order & 11.44
\end{tabular}
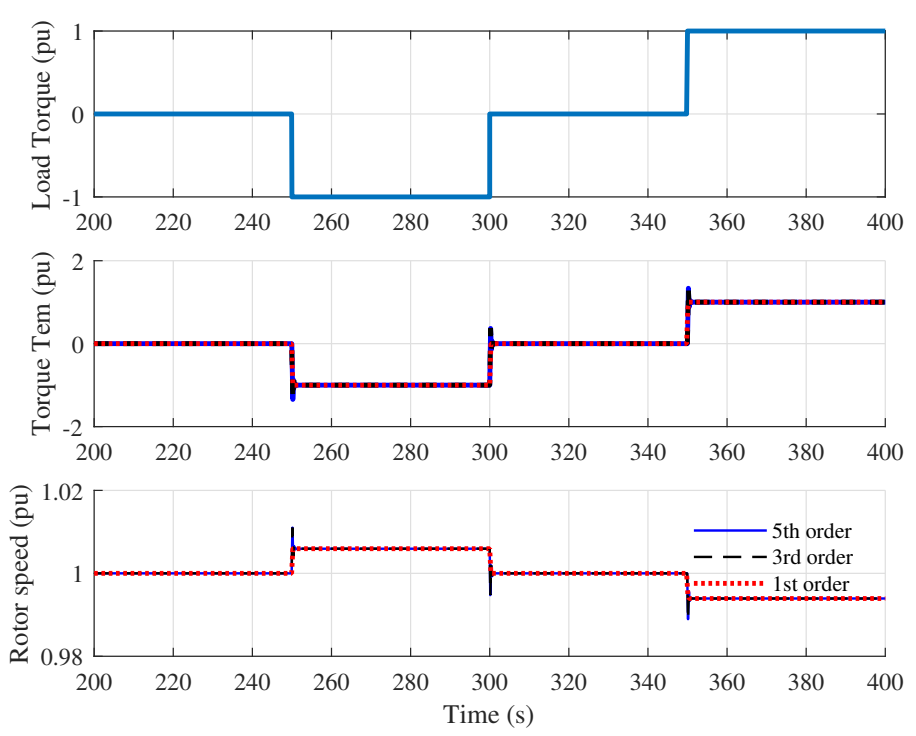

Fig. 2. Response of reduced order machines to step changes in load torque

\section{B. Performance as VFT}

The models are then re-purposed for use as a VFT. New parameters are specified in table III which are in agreement with previously used parameters for VFT studies [6]. 


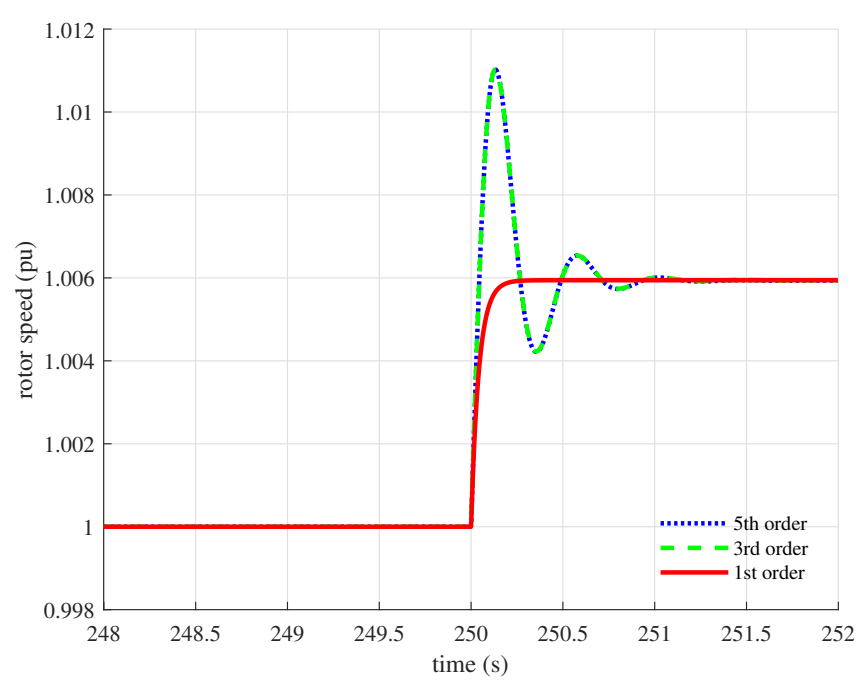

Fig. 3. Close-up of a transient

TABLE III

TYPICAL VFT PARAMETERS

\begin{tabular}{l|l|l|l} 
Parameter & Value & Parameter & Value \\
\hline$S_{b}$ & $100(\mathrm{MW})$ & $L_{l s}$ & $0.1(\mathrm{pu})$ \\
$V_{s}, V_{r}$ & $23(\mathrm{kV})$ & $L_{l r}$ & $0.1(\mathrm{pu})$ \\
$r_{s}$ & $0.005(\mathrm{pu})$ & $L_{m}$ & $10(\mathrm{pu})$ \\
$r_{r}$ & $0.005(\mathrm{pu})$ & $H$ & $25(\mathrm{~s})$
\end{tabular}

The first simulation connects two synchronous grids, both operating at a frequency of $50 \mathrm{~Hz}$ and tracks power flow through the VFT as a function of the applied torque. In this test, a negative torque of $-0.5 \mathrm{pu}$ is applied at $t=80 \mathrm{~s}$ and then removed at $t=160 \mathrm{~s}$ before a positive torque of $0.5 \mathrm{pu}$ is applied at $t=240 \mathrm{~s}$. The results are given in fig. 4 and 5. Fig. 4 shows that the 5 th and 1 st order models closely follow the power demand and are in good agreement with one another. $0.5 p u$ torque facilitates the transfer of $0.5 p u$ power between the two grids as expected. Fig. 5 however, shows the inaccuracies of the 3rd order model with large oscillations around the set point that reach $\pm 0.2 p u$. These indicate very little natural damping given the small values of their resistive elements.

Table IV gives the computation times for each VFT model. The 3rd has been omitted due to its unsuitability in this application, however the 1st order model shows an $18 \%$ decrease in elapsed time compared to the fifth order model.

TABLE IV

SIMULATION TIMES OF VFT MODELS

\begin{tabular}{l|l} 
Model & Time $(\mathrm{s})$ \\
\hline 5th Order & 18.83 \\
1st Order & 15.39
\end{tabular}

\section{Asynchronous Power Transfer}

The two grids are then put out of synchronism to test the stability of the model under asynchronous power transfer. Traditionally, VFT's are installed to link networks which

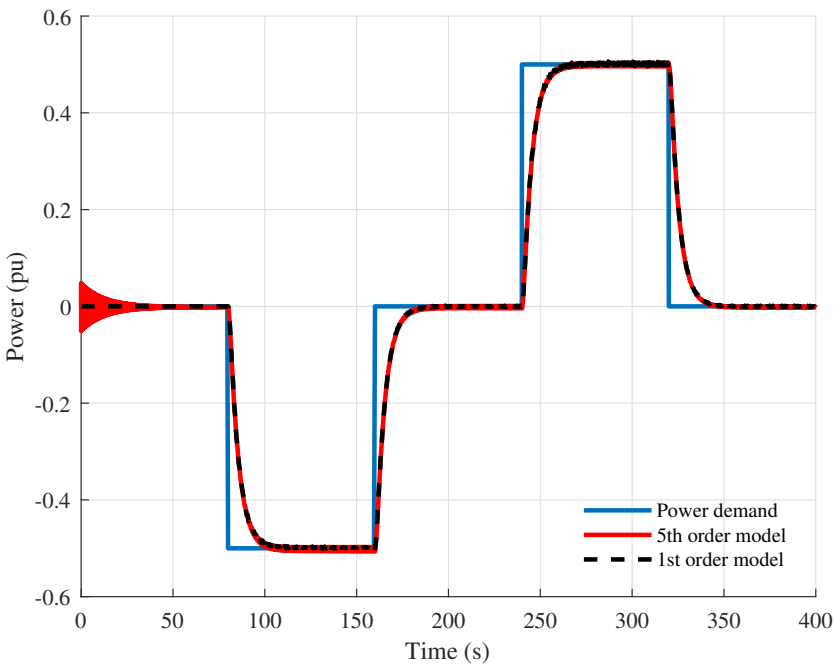

Fig. 4. Power transfer between synchronous networks using 5th and 1st order model

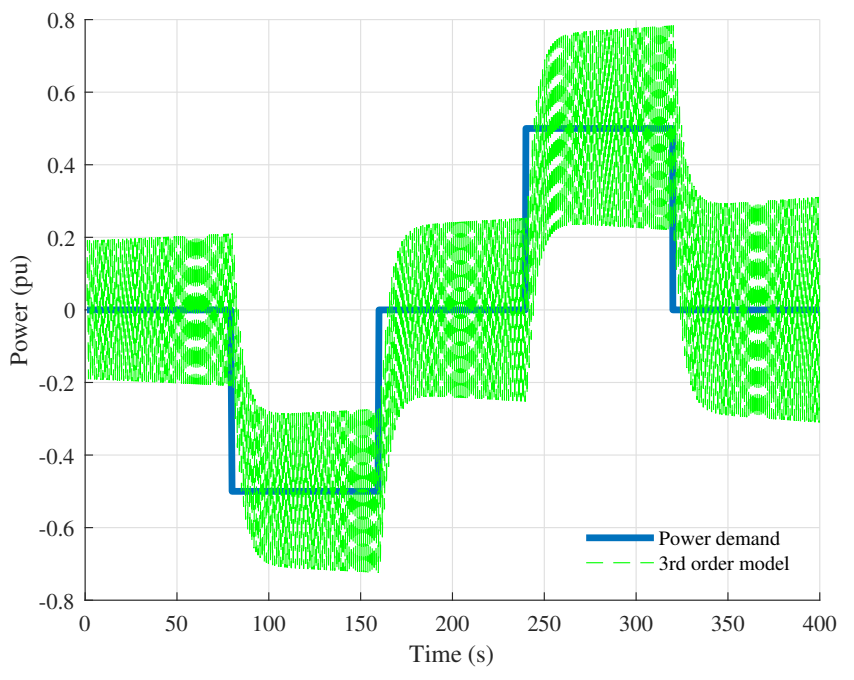

Fig. 5. Unsuitability of 3rd order model for the parameters in table III

only have slightly drifting frequencies, however to test the capabilities of this model, a more ambitious frequency differential is employed. The stator side network is set to $50 \mathrm{~Hz}$ while the rotor side is set at $60 \mathrm{~Hz}$ simulating a possible interconnection between asynchronous countries [9].

At steady-state, the rotor spins at a speed proportional to the slip as shown in (2) which in this instance is at $0.2 p u$. The 1st order model speed characteristic is shown in fig. 6 . The output of this model experiences some noise; nevertheless the error margins are quite small. For visualization purposes, a filtered version of the rotor speed output for the 1st order model is also presented in fig. 6 . The resulting speed characteristic is shown to follow the expected trajectory. The same torques are then applied as in the previous experiment showing that the 1st order model is fully capable of simulating a VFT under an asynchronous power transfer. 


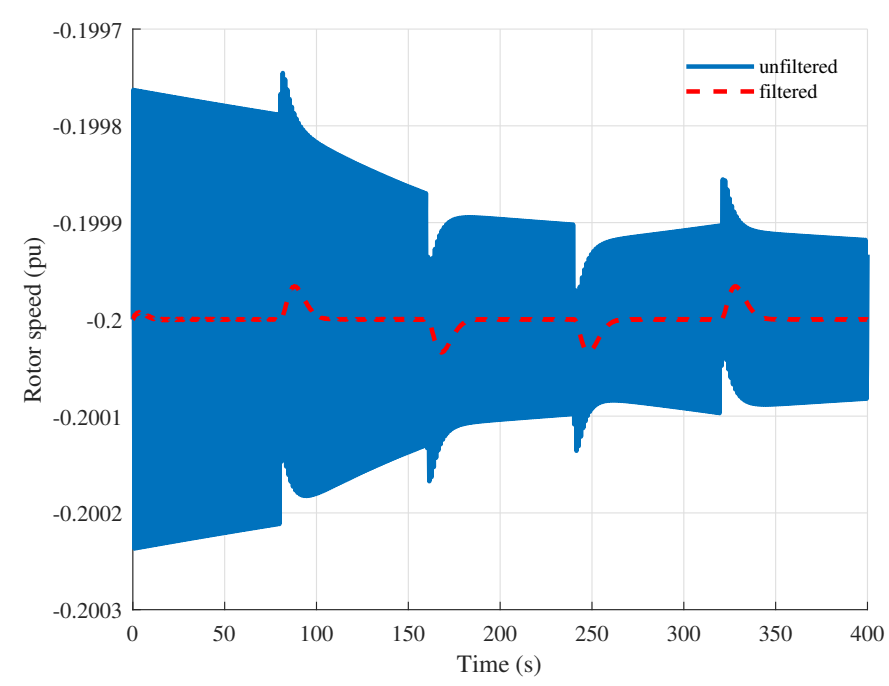

Fig. 6. Rotor speed output for the 1st order model. In blue unfiltered output, in dotted red filtered output.
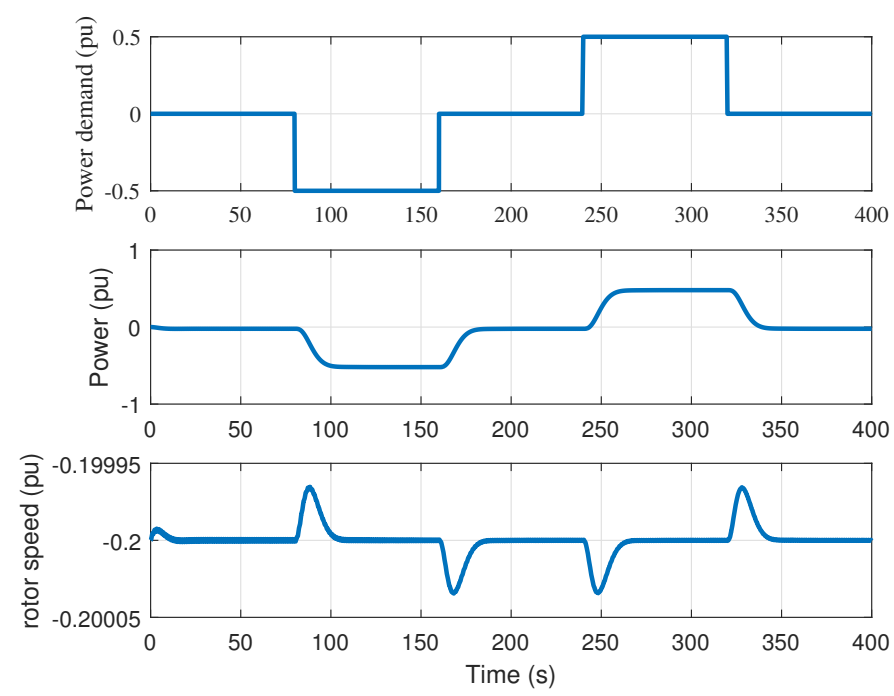

Fig. 7. 1st order model power transfer characteristics

\section{CONCLUSions}

This work has explored the suitability of reduced order models for induction machine simulations and has also highlighted some strengths and weaknesses of the various models when applied to VFT power transfer studies. A new third and first order method have been presented which contain all of the information relating to the frequency of the stator. These models have also been designed to avoid algebraic loops within simulation.

All three models are effective when simulating an induction machine but for the VFT parameters specified in table III the third order model becomes unstable. The low resistance characteristics of the VFT make the 3rd order model incompatible when used with the data in table III, however for more naturally damped values the 3rd order may be able to function correctly.

By neglecting the electrical transients of the rotor, the first order model does well at capturing key information relating to rotor speed and electromagnetic torque without losing accuracy with the added advantage of a reduced computational effort.

The first order model has also been shown to be effective at simulating a VFT when interconnecting two networks. When the networks are in synchronism, the model shows good power tracking when compared to the already well documented fifth order model thus demonstrating its usefulness in power flow studies. When the two networks are at different frequencies, the rotational speed of the rotor accurately matches up to the values predicted by the literature and when a power order is sent via an applied torque the first order model tracks the demand well.

\section{REFERENCES}

[1] General Electric, Variable Frequency Transformer http://www.gegridsolutions.com/powerd/vfts.htm

[2] B. Ambati, P. Kanjiya, V. Khadkikar M. Moursi and J. Kirtley A Hierarchical Control Strategy With Fault Ride-Through Capability for Variable Frequency Transformer. IEEE Transactions on Energy Conversion, Volume: 30, Issue: 1, March 2015

[3] A. Merkhouf, P. Doyon, S. Upadhyay. Variable Frequency Transformer Concept and Electromagnetic Design Evaluation. IEEE transactions on energy conversion, vol. 23, no. 4, December 2008

[4] L. Contreras-Aguilar, N. Garca, M. A. Islas-Martnez R. Adame-Ortiz. Implementation of a VFT Model in PSS/E Suitable for Power Flow and Transient Stability Simulations. 2012 IEEE Power and Energy Society General Meeting

[5] D. McNabb, D. Nadeau, A. Nantel, E. Pratico, E. Larsen, G. Sybille. Transient and Dynamic Modeling of the New Langlois VFT Asynchronous Tie and Validation with Commissioning Tests. International Conference on Power Systems Transients 2005

[6] R.J. Piwko, E.V. Larsen, C.A. Wegner. Variable Frequency Transformer A New Alternative For Asynchronous Power Transfer. Inaugural IEEE PES 2005 Conference and Exposition in Africa Durban, South Africa, 11-15 July 2005

[7] B. Bagen, D. Jacobson, G. Lane and H. M. Turanli. Evaluation of the Performance of Back-to-Back HVDC Converter and Variable Frequency Transformer for Power Flow Control in a Weak Interconnection. 2007 IEEE Power Engineering Society General Meeting

[8] J.B. Ekanayake, L. Holdsworth, N. Jenkins. Comparison of 5th order and 3rd order machine models for doubly fed induction generator $(D F I G)$ wind turbines. Electric Power Systems Research 67 (2003) 207-215

[9] T.M. Aljohani, A.M. Alzahrani. The Operation of the GCCIA HVDC Project and Its Potential Impacts on the Electric Power Systems of the Region. International Journal of Electronics and Electrical Engineering Vol. 2, No. 3, September, 2014

[10] C. Cai, P. Ju and Y. Jin. Novel Simplified Model for Asynchronous Machine with Consideration of Frequency Characteristic. Journal of Applied Mathematics Volume 2014, Article ID 701964

[11] C.M. Ong. Dynamic Simulation of Electrical Machinery. Ch.6. 